\title{
Theories of spatial representations and reference frames: What can configuration errors tell us?
}

\author{
Ranxiao Frances Wang
}

Published online: 8 May 2012

(C) Psychonomic Society, Inc. 2012

\begin{abstract}
The issue of reference frame is central to theories of spatial representations. Various classifications have been made for different types of reference frames, along with prototypical research paradigms to distinguish between them. This article focuses on the configuration error paradigm proposed by Wang and Spelke (Cognition 77:215$250,2000)$ that has been used to examine the nature of the spatial representations underlying object localization during self-movement. Three basic models of spatial memory and spatial updating are discussed, as well as the assumptions behind the configuration error paradigm, to distinguish between static representations, such as the traditional allocentric cognitive map and the egocentric snapshots, and dynamic representations, such as the egocentric updating system. Recent experimental findings are reexamined and shown to be consistent with multiple models, among which the egocentric-updating-and-reload model with an enduring egocentric component provides the simplest interpretations.
\end{abstract}

Keywords Configuration error - Reference frame .

Egocentric updating $\cdot$ Cognitive map $\cdot$ Snapshot

\section{Introduction}

Spatial representations are essential to the survival of humans and nonhuman animals. It has been shown that many animals, including insects such as ants and bees,

\footnotetext{
R. F. Wang $(\bowtie)$

Department of Psychology, University of Illinois

at Urbana-Champaign,

Room 533, 603 E. Daniel St,

Champaign, IL 61820, USA

e-mail: francesw@cyrus.psych.illinois.edu
}

possess some form of spatial knowledge of their environment to guide their navigation during daily foraging activities (e.g., Cartwright \& Collett, 1982; Griffin \& Etienne, 1998; Lehrer \& Collett, 1994; Mallot \& Gillner, 2000; Wang $\&$ Brockmole, 2003). How animals represent spatial locations and navigate has engaged much empirical investigation and theoretical debate. For example, what type of information do animals represent? Can they learn and use landmarks or other features of the environment? Do they encode metric spatial relations or just response patterns? And how do they keep track of their spatial relations with the environment as they move around?

One of the central questions involving spatial representations is the type of reference frames used to encode spatial information. Mathematically, a spatial reference frame contains two basic elements. One is the reference point, or the origin. The other is the reference direction, or the axes. With these two elements and the unit distance and angle, any location in space can be specified with a unique set of coordinates (Klatzky, 1998; Wang, 2003). Different types of reference frames can be defined on these two elements, according to either the reference direction or the reference point. For example, on the basis of the reference point, an animal may encode either the object-to-self relationship (egocentric) or the object-to-object relationship (allocentric). On the basis of the reference direction, the reference frame can be defined by the viewer's heading (relative reference), by the orientation of an external object (intrinsic reference), by the earth's northsouth-east-west (absolute reference), and so on.

Various methods have been developed to classify different types of spatial representations, many of which were based on the reference frames used to encode spatial information. For example, three classical approaches have been widely used to divide spatial representations into two main categories - that is, egocentric versus allocentric representations. The first 
approach concerns the distinction between route and survey knowledge. Route knowledge consists of spatial information restricted to the path that connects the origin and the goal, which may include a list of path segments, intersections, and the position of landmarks on or near the path. In contrast, survey knowledge is generally conceived of as maplike representations that contain information about the spatial relationship among significant locations. Because route knowledge is obtained from the direct perceptual experience during navigation from a perspective of the navigator, it is often considered egocentric. In contrast, survey-type knowledge is not directly available from perceptual experience for land-bound animals and needs to be constructed through additional spatial processing and integration into a mental map from a bird's-eye perspective external to the navigator. Thus, survey knowledge is often referred to as allocentric (e.g., Gould, 1986; Landau, Spelke, \& Gleitman, 1984; Maguire et al., 2003; Tolman, 1948).

A classical method used to examine whether an animal forms a mental map of the environment is the novel-shortcut test. This research paradigm provides a test on whether an animal's spatial learning is restricted to specific routes or is more general, survey-type knowledge (i.e., mental maps). In a typical procedure of this paradigm, animals are trained to go to feeder A and feeder B from home separately but are never allowed to travel between A and B directly. During the test, the animals that are on an outbound trip to $B$ are transported to A. If, upon being released from $\mathrm{A}$, the animals are able to go directly to $\mathrm{B}$ on this never-learned route, it is concluded that the animal has built a mental map of the environment that allows the animal to infer new spatial relationships.

The second approach concerns the distinction between landmark-based versus snapshot representations. Snapshots are generally conceived of as spatial memories of a scene that resemble the retinal images. In contrast, landmarkbased representations encode target locations relative to the special features in the environment (landmarks). Thus, when an animal uses snapshot memory to retrieve a target, it should search by matching the retinal images of the scene to the stored one. When retrieving a target using landmarkbased representations, on the other hand, an animal should search at locations that match the description of the relationship between the target and the landmark.

The research paradigm commonly used to test snapshot versus landmark-based spatial knowledge is the landmarkshift test. In this paradigm, the animal typically learns a target location (such as a feeder) with a number of landmarks nearby forming a specific configuration. During the test, the feeder is hidden, and the landmarks are changed (e.g., in size, orientation, etc.) or moved around and rearranged in various ways. The animal's searching patterns are recorded to see whether it uses the landmark information to locate the target and, if so, which information is used (e.g., the distance, orientation, proximity, perceptual saliency, etc.) and how (e.g., the selection and weighting of conflict information). If an animal searches in a location that has the best match in retinal image of the surrounding (e.g., searching at twice the distance away when the landmark doubles its size), a snapshot representation is used for target localization, and the representation is considered egocentric (Collett \& Cartwright, 1983; Collett \& Lehrer, 1993; Collett \& Rees, 1997). On the other hand, if the animal searches for the hidden target in a certain relationship to the location and orientation of the landmark cue(s) (e.g., always searching $3 \mathrm{~m}$ from the cylinder in the direction of the triangle, regardless of where the two landmarks are placed and how far apart they are), it is often taken as evidence that the animal uses the landmarks as its reference to locate the target, and the spatial representations are thus considered allocentric in nature (e.g., Burgess, 2006; Burgess, Spiers, \& Paleologou, 2004; Gleason \& Rothblat, 1994).

The third approach concerns the distinction between viewdependent and view-invariant representations. Viewdependent representation contains spatial information encoded relative to a specific viewpoint, while viewinvariant representation contains spatial coding that does not depend on a specific viewpoint. The research paradigm commonly used to test the view-dependent versus view-invariant representations is the perspective-taking test. In a typical procedure, an observer studies an array of targets from specific perspective(s). During the test, the observer is asked to make spatial judgments from various perspectives either familiar (same as the study perspective) or novel. The tasks include scene recognition (judging whether a layout seen from the testing perspective is the same or different), reproduction (placing the target objects at their original positions while the observer is standing/sitting at a different location), and judgment of relative directions (indicating the direction of one object relative to another from a test perspective). Performance variations among the test perspectives are often interpreted as evidence that the spatial memory representation is view dependent, while constant performance across different test perspectives indicates view-invariant representations. An advantage of the studied viewpoint is often interpreted as evidence for egocentric representations, while both view invariance and advantages of perspectives defined external to the observer (e.g., the axes of the array itself or environmental axes) are considered evidence for allocentric reference frames (e.g., Endress \& Wood, 2011; Mou \& McNamara, 2002; Shelton \& McManara, 2001; Wang, 2007).

A large literature in spatial memory and navigation across many species, both behavioral and neural physiological, has employed some variants of these paradigms. These approaches all have focused on the form of long-term memory. However, it has been pointed out that a well-known process found in most navigating animals - that is, path integration (and spatial updating in general) - can provide 
alternative explanations for many spatial behaviors without evoking the supposed type of spatial representations. For example, novel shortcut can be achieved through path integration without constructing cognitive maps (survey knowledge) (Bennett, 1996). Similarly, Simons and Wang (1998; Wang \& Simons, 1999) showed that view-invariant scene recognition may be achieved by spatial updating without relying on view-invariant spatial coding. These findings call for a different theoretical approach in analyzing spatial representations and reference frames that takes into account the dynamic processes of spatial updating both at the conceptual level and in designing and interpreting experimental paradigms.

\section{The configuration error paradigm}

A different way to categorize different types of reference frames was proposed by Wang and Spelke (2000, 2002, 2003) based on whether the spatial coding needs to change when an observer moves. In this approach, any reference frame that concerns the observer's position and/or heading is considered egocentric, because the validity of the spatial information encoded in such reference frames depends on the observer. In contrast, an allocentric reference frame is constructed entirely on information external to the observer, and therefore the validity of the spatial coding is independent of both the observer's position and heading. According to this classification, all mixed representations (e.g., self-to-object relations encoded in a north-south directional frame, or object-to-object relations encoded in a directional frame determine by the animal itself) are all classified as egocentric representations. This definition is especially suitable for the study of navigation, where some spatial relationships keep changing and need to be updated, while others remain constant as an animal moves through a stable environment, and whether and when the spatial information is valid is critical for successful spatial localization during navigation. Thus, this classification of spatial representations based on their dependence on observer movements was adopted by Wang and Spelke (2000) in the research paradigm developed to examine the nature of spatial representations and processes in navigation.

To examine the nature of spatial representations for localizing targets when a navigator moves through an environment, Wang and Spelke (2000) proposed a configuration error paradigm. Participants learned the locations of multiple target objects and then pointed to these objects while blindfolded under different conditions. They were tested after a simple body turn, after continuous turning for $1 \mathrm{~min}$ with a directional cue present to keep them oriented, after being disoriented by continuous turning without any directional cues, or after being disoriented and then reoriented by a directional cue. Moreover, corners of a room were compared with individual objects forming the same geometric configurations, in either a rectangular shape or an irregular shape. The primary interest was to examine whether people's pointing responses to multiple targets preserve the same internal consistency across various experimental conditions and target types.

Wang and Spelke (2000) separated people's response errors into two independent components, the heading error and the configuration error, and used the configuration error to compare the consistency in the internal relations of people's pointing responses across conditions. The heading error was defined as the mean of the signed errors for each target and indicates the overall rotation of all targets. Heading errors primarily stem from people's misperception of their heading. The configuration error was defined as the standard deviation of the signed errors to each target. ${ }^{1}$ If people's responses preserve the internal relationship among different targets, the errors made to each target should be the same, and the configuration error should be zero. In contrast, if the configuration error is larger than 0 , the angular configuration of the responses is different from the correct target configuration. The larger the configuration error, the more dissimilar the two configurations. Thus, the configuration error provides a measurement of the deviation in the configuration of a set of responses.

Using this paradigm, Wang and Spelke (2000) showed that the configuration errors increased after people were disoriented, as compared with the oriented turning conditions, and did not recover even when people were disoriented and then reoriented by a directional cue. These results ruled out the alternative explanations of the disorientation effect as a result of physical activity, difference in perspective, and uncertainty of one's self-orientation. Moreover, the increase in configuration errors after disorientation seemed to be specific to object arrays and did not occur for corners of a room, either in a rectangular shape or an irregular shape.

These results were interpreted as supporting a transient egocentric representation that is updated as people move and rejecting the traditional enduring allocentric cognitive map theory. ${ }^{2}$ Since then, a number of research groups have

\footnotetext{
${ }^{1}$ Some researchers refer to the heading error as the constant error and the configuration error as the variable error. However, variable errors in the literature typically refer to the within-target variability, which corresponds to the pointing error in Wang and Spelke's (2000) paradigm and has a different theoretical meaning than the configuration error, which measures the intertarget variability.

${ }^{2}$ Here, the enduring and transient representations are defined with respect to the observer movements. An enduring representation may change (e.g., deteriorate or distort) over time and will still be considered enduring as long as it is independent of the self -movements. In addition, the distinction between enduring and transient representations does not necessarily map to long-term memory and working memory, respectively. For example, it is conceivable that an enduring representation (e.g., an egocentric snapshot) is maintained in working memory without being updated. However, a transient, dynamic representation in long-term memory is less plausible.
} 
employed the configuration error paradigm to study the reference frames in human spatial memory and processes (e.g., Chan, Chang, \& Sun, 2007; Holmes \& Sholl, 2005; Mou, McNamara, Rump, \& Xiao, 2006; Sargent, Dopkins, Philbeck, \& Chichka, 2010; Sargent, Dopkins, Philbeck, \& Modarres, 2008; Waller \& Hodgson, 2006; Xiao, Mou, \& McNamara, 2009). However, many of the theoretical interpretations of the experimental results are problematic and have generated much confusion on the issue of spatial reference frames. To clarify these theoretical confusions, a careful examination of the assumptions behind various theories of spatial representations is needed.

\section{Models of spatial representation systems}

\section{Egocentric snapshot}

Traditional models of spatial representations can take two basic forms. Egocentric representations encode the location and orientation of objects relative to the animal itself, using reference frames defined by the animal's position and/or heading (Fig. 1a). It is generally accepted that the spatial information directly supplied by perception is egocentric, and egocentric information is required to perform actions. However, the spatial information encoded in egocentric representations is correct only when the animal is located at the same position and facing the same direction. Thus, egocentric representations are generally considered to be the most primitive form of spatial knowledge, which is easier to acquire and can be applied directly for action guidance, but to be inflexible and limited in their usefulness.

Egocentric snapshot representations can take many different forms. For example, the snapshot representation originally implies that the representation is 2-D and image-like, such as

Fig. 1 An illustration of an egocentric snapshot model (a), an allocentric cognitive map model (b), and an egocentric updating model (c). The thin solid arrows indicate what relationships are represented, the double solid arrow indicates the reference frame in which spatial information is coded, and the dashed arrows indicate what is updated the visual snapshots shown to underlie navigation in honeybees (Collett \& Cartwright, 1983; Collett \& Lehrer, 1993). The spatial information contained in these snapshots is correct only for the position at which they were taken and, therefore, is egocentric in nature by definition. However, the concept does not have to be restricted to retinal images and can be generalized as egocentric snapshot representations, which include all types of spatial representations that capture the state of the environment at a given moment (i.e., a "snapshot" of the environment) from the perspective of the observer (i.e., "egocentric").

For example, an animal may encode the location of prominent environmental features from its study perspective, each represented as a vector defined by a direction and distance (Fig. 1a). These vectors can be expressed in polar coordinates with the heading of the observer as the directional reference (e.g., the candle is $12 \mathrm{~m}$ away at $-30^{\circ}$, the mailbox is $8 \mathrm{~m}$ away at $45^{\circ}$, etc.) or with an external direction such as north or the direction of the candle as the reference (e.g., the mailbox is $8 \mathrm{~m}$ away at $75^{\circ}$ ). These vectors can also be expressed in Cartesian coordinates, with either the origin or the direction of the axes anchored on the observer, while the other one could be defined by external environmental features. Moreover, object-to-object relationships that are expressed using directional reference anchored on the observer are also considered egocentric, because the validity of such spatial information depends on the observer and needs to change as the observer moves. For example, the position of the candle relative to the mailbox can be expressed as $9 \mathrm{~m}$ at $-50^{\circ}$ relative to the heading of the observer (Fig. 1a).

\section{Allocentric cognitive map}

In contrast, allocentric cognitive maps encode the spatial relationship among features of the external environment using

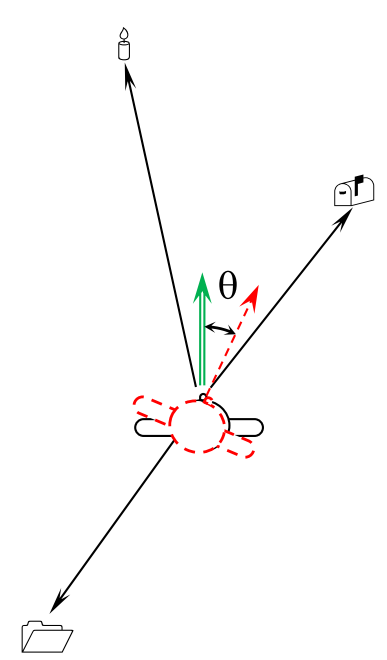

a

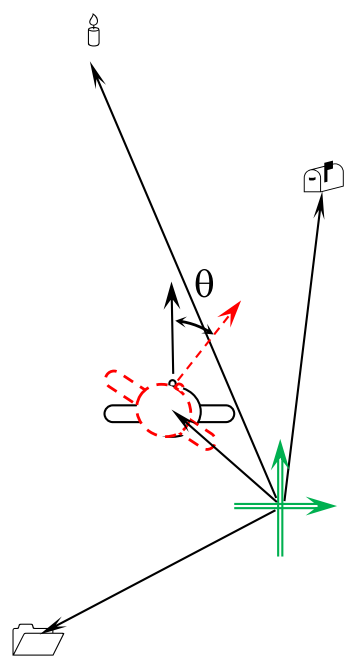

b

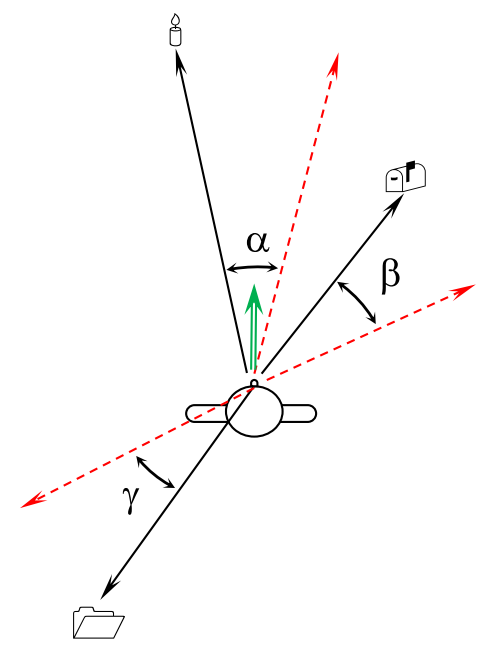

C 
externally defined reference frames and, therefore, are completely independent of the animal (Fig. 1b). Allocentric cognitive maps have been considered the most advanced form of spatial knowledge, and there has been considerable debate on whether specific animal species have achieved this cognitive benchmark (e.g., Bennett, 1996; Easton \& Sholl, 1995; Gallistel, 1990; Mou \& McNamara, 2002; O'Keefe \& Nadel, 1978). Allocentric spatial information cannot be used to guide actions directly, however. In order to use an allocentric map, a map lookup process has to convert the spatial information in the map into the egocentric coordinates by using a separate representation about the animal's position and heading in the cognitive map's reference frame. An allocentric cognitive map model posits three basic premises: (1) an enduring memory representation that encodes allocentric spatial information, which constitutes the animal's spatial knowledge about its environment; (2) a representation of the animal's location and heading using the reference frame of the cognitive map, which does not contain information about the environment by itself and can change over time and be updated as the animal moves; (3) a lookup process that integrates the two representations above and converts the spatial information in the cognitive map into the animal's egocentric reference frame to perform various actions. This lookup process is sometimes described as the alignment between the egocentric and the allocentric reference frames (e.g., Easton \& Sholl, 1995).

The standard model Allocentric cognitive maps can also take many forms. For example, a spatial representation that specifies the distance and direction of each object relative to an external coordinate system (Fig. 1b) is a standard form of allocentric cognitive map. These vectors can be expressed either in polar coordinates (e.g., the candle is at $\left[12 \mathrm{~m},-30^{\circ}\right]$ ) or in Cartesian coordinates (e.g., the candle is at $[-6 \mathrm{~m}, 10.4 \mathrm{~m}])$. In these models, the position and heading of the observer are typically also expressed in the same reference frame (e.g., the observer is at $\left[4 \mathrm{~m},-60^{\circ}\right]$ or at $[-3.5 \mathrm{~m}, 2 \mathrm{~m}]$ facing $45^{\circ}$ ). This piece of information is not part of the allocentric cognitive map and changes as the observer moves.

The interobject relationship model Another common form of allocentric cognitive map is the object-to-object relationships. These models encode pairwise direction and distance relationships among a set of objects (e.g., Mou \& McNamara, 2002). The direction of one object relative to another may be defined by the geographical directions (e.g., the ash tree is $12 \mathrm{~m}$ north of the rock) or by the direction of a landmark (e.g., the ash tree is $12 \mathrm{~m}$ downhill from the rock). ${ }^{3}$ Similarly, these relationship

\footnotetext{
${ }^{3}$ When the reference direction is defined by the observer (e.g., the facing direction during studying), the representation becomes egocentric according to the present theoretical framework, because such spatial coding is no longer valid as the observer turns.
}

vectors can also be expressed in Cartesian coordinates, with both the origin and the axes defined external to the observer. Note that the number of pairwise interobject relationships increases quadratically as the number of objects increases, and most of the information is redundant (Meilinger, 2008; Wang, 2007). One solution to avoid the redundancy is to encode only a subset of these relationships - for example, by identifying a special reference object and encoding only spatial relations to that reference object. In this case, the model becomes essentially the same as the standard form of allocentric cognitive map. Another way to reduce the redundancy is to encode only the distance information, and from a full set of pairwise interobject distance data, the directional relationship among the objects can be recovered. It is also possible to encode the directional information only, and the distance relationship may be recovered up to a scale factor. The number of spatial relations still increases quadratically with the number of objects for the pure-distance or pure-direction models, however, and again most of the information is redundant.

In the interobject relationship models, the position of the observer also needs to be specified, either by its relationship to a single reference object or between the observer and all other objects. The former case is the same as the additional piece of information in the standard form of the allocentric cognitive map model. The latter case, however, is essentially a complete egocentric snapshot representation by itself. When this representation is available, the interobject relationships in the allocentric cognitive map are completely redundant, and the representation is effectively a dual representation.

Spatial localization using the enduring representations

Although these two traditional forms of spatial representations are drastically different, they make identical predictions on the effects of spatial updating and disorientation in the configuration error paradigm. Both the egocentric snapshot and the allocentric cognitive map are enduring representations and predict constant configuration errors throughout spatial updating and disorientation. For the allocentric cognitive map mod$\mathrm{el}$, because the map itself is maintained independently of the self-position representation, the only process that can alter the intertarget relationship is the lookup process. Thus, if one makes the reasonable assumption that the lookup process itself is not affected by one's knowledge of self-position and heading, the configuration errors of multiple target responses should remain constant. ${ }^{4}$

\footnotetext{
${ }^{4}$ The lookup process may introduce configuration errors if the selfposition and heading are uncertain and vary during the lookup process. This artifact may be subtracted from the raw configuration errors based on the pointing errors to reveal the genuine configuration error effects, or using experimental conditions that remove the uncertainty of self-position and heading, such as reorientation. Both approaches have been employed in the literature (e.g., Waller \& Hodgson, 2006; Wang \& Spelke, 2000).
} 
Similarly, an egocentric map can be used in the same manner as an allocentric map is used (Fig. 1a). That is, a reorientation process that aligns an animal's current position and heading relative to its position and heading during the encoding stage can transform the old egocentric codes to a set of new ones. Therefore, if an animal takes a "snapshot" of the egocentric relations at the beginning of a trip and keeps track of its movements since its original heading and position, it can look up the original coordinates of the targets in the egocentric map and compute their current coordinates whenever it needs to. This process is mathematically the same as the lookup process in the use of an allocentric map. Thus, any alteration of the configuration among objects in the egocentric map has to come from the lookup process, and the configuration errors should be a constant if one makes the same assumption that the precision of the lookup process is independent of one's self-position knowledge.

Egocentric updating and properties of dynamic representations

In the egocentric updating model proposed by Wang and Spelke (2000), the nature of the spatial-updating process was fundamentally different from that of the two traditional models, in that the updating system is not a complementary process used to support the lookup process but, instead, constitutes an animal's spatial knowledge of the environment. Thus, the updating system maintains a transient, dynamic map all by itself (Fig. 1c). Four basic assumptions were made about an egocentric updating system: (1) The representation encodes spatial information about the environment that depends on the animal's position and/or heading (and therefore, is egocentric by definition); (2) this spatial coding needs to be updated as the animal moves on the basis of the perceptual information of its selfmovements; (3) in each step, the updating process computes the new egocentric map, which replaces the old one; (4) actions can be performed directly using this dynamic representation without any further transformations or processes.

On the basis of these basic assumptions of the egocentric updating model, the maintenance of the configuration among targets depends on the integrity of the updating process. If one makes the reasonable assumption that the updating process of multiple targets is performed individually and not noise-free, one would expect the configuration errors to increase as the updating progresses, especially when disruption of spatial updating occurs. However, disorientation does not necessarily abolish the dynamic representation completely; therefore, the degraded representation that remains in the spatial-updating system can potentially be used and provide nonrandom performance after disorientation (Wang \& Spelke, 2000). This hypothesis will be referred to as the egocentric-updating-withresidual hypothesis.
Note that the independence assumption necessary for the prediction of increase in configuration error is not specific to the egocentric updating model. To be more specific, the independence assumption contains two assumptions: (1) The operation (e.g., updating) is performed on each object individually (individuality assumption); and (2) the operation has noise (noise assumption). The individuality assumption is considered a necessary consequence of individual spatial coding for each target, and it applies to all models (egocentric updating, egocentric snapshot, and allocentric map). That is, because each object is represented by an individual vector, any operation on these vectors has to be performed individually for each object. This is not only true for egocentric updating, but also true for the lookup process of the allocentric cognitive map and the egocentric snapshot, where each object vector is assumed to be transformed individually. Similarly, the noise assumption is also common to all models. Because these assumptions are common to all models, they were not listed as the basic assumptions for the individual models.

From the mathematical point of view, the key assumption that differentiates the egocentric updating system and the enduring representations is assumption (3) - that is, the replacement assumption. For the enduring representation models, the original copy of the representation is retained; therefore, regardless of how many times the transformation/ lookup process is performed, the next operation is not affected. Therefore, the size of configuration error is determined solely by the noise level in the present operation, regardless of what happened before that during the interval. In contrast, for the egocentric updating model, the original copy is discarded as soon as the updating process starts, and each representation is replaced by the next; thus, the configuration errors propagate from one step to the next and accumulate over time. In other words, the size of the configuration error is, in general, primarily determined by what happened prior to the testing. As a result, the egocentric updating model predicts an increase in configuration error after disorientation because the disruption is carried over to the postdisorientation performance, while the enduring representation models predict a constant configuration error because each lookup process is based on the original copy and, therefore, equally accurate, assuming that the noise level during testing before and after disorientation is comparable.

\section{The place cell theories}

The hippocampal place cells first discovered in rodents show certain intriguing behavior that has typically been taken as evidence of allocentric cognitive maps in the brain. These neurons have place fields that correspond to specific regions of space, and the neuron fires whenever the animal 
passes through its place fields, generally independently of the animal's heading (McNaughton, Knierim, \& Wilson, 1995; O'Keefe \& Nadel, 1978; O'Keefe \& Speakman, 1987; Wilson \& McNaughton, 1993). A population of such neurons (i.e., place cells) can map out the environmental space and collectively signal the allocentric position the animal occupies at each moment. Thus, place cells have been considered the neural implementation of an allocentric cognitive map.

A careful examination of the place cell behavior shows that this neural "cognitive map" is not the real allocentric cognitive map that encodes the spatial information of environmental features in an external reference frame (Wang, 2003). An allocentric cognitive map is a static representation and does not change as the animal moves, while the place cell system changes dynamically as the animal moves. One possibility is that the place cell system functions like the "where I am" system that complements an enduring representation, which can be either an egocentric snapshot or an allocentric cognitive map. As was discussed above, the reference frame used in the "where I am" system should be matched with the reference frame used in the enduring representation it is paired with. Thus, two models of the place cell + enduring representation systems are possible. First, the place fields of the place cells are defined relative to the external environment, which are paired with an allocentric cognitive map encoded in the same reference frame. Alternatively, the enduring representation may be an egocentric snapshot, and the place cell system is defined relative to the position and heading of the animal itself when the snapshot is taken. ${ }^{5}$ Thus, the presence of place cells does not necessarily mean the existence of an allocentric cognitive map.

It is also possible to conceive of the place cells as directly representing spatial knowledge of the environmental features. The hippocampus is shown to be important for relational learning (e.g., Eichenbaum \& Cohen, 1988; Eichenbaum, Otto, \& Cohen, 1992). Thus, place cells could be responding to multiple egocentric relationships of various types. For example, a neuron that encodes the "and" relationship between two egocentric distances (e.g., "the cylinder is $5 \mathrm{~m}$ from me" and "the triangle is $2 \mathrm{~m}$ from me") will fire at given locations in space, regardless of the animal's heading. Similarly, a neuron responding to the difference relationship between two egocentric directions (e.g., "the direction of the cylinder is $30^{\circ}$ smaller than the direction of the triangle") will be invariant to the animal's heading but change as the animal

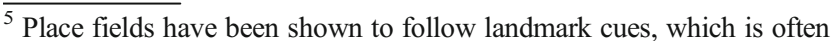
taken as evidence of allocentric representations according to the landmark shift paradigm. However, these findings can also be accommodated by the egocentric snapshot model, if the animal uses the cues to reorient/ relocate itself, which effectively aligns the egocentric space (and the place fields defined within it) with the cues. See the Theoretical Interpretations section for more discussion on the reorientation process.
}

moves. ${ }^{6}$ The targets may be any environmental features, including parts of an object such as the two vertical edges of a cue card, the center of the arena, and so forth, and those currently out of view due to occlusion, behind the animal or in the dark. When the targets are invisible, their egocentric positions can be updated continuously, so that these neurons may still respond at the appropriate locations without directly perceiving the targets. Moreover, different neurons can encode different targets and different relationships; therefore, they may respond differently to a given manipulation. For example, it was shown that some place cells have place fields that rotate along with a moving cue card, while others do not (e.g., Knierim, Kudrimoti, \& McNaughton, 1995). These results are consistent with the hypothesis that some neurons encode features of the cue card and, therefore, change their place fields as the card moves, while others encode features of the stable environment and, therefore, their place fields stay in the original location. When the place cell system is interpreted as spatial coding of the locations of environmental features, this spatial representation is dependent on the observer because the neurons' activities change as the animal moves; therefore, by definition, it is a dynamic egocentric representation. ${ }^{7}$ Overall, a place cell system is actually consistent with all three models: the egocentric snapshot model, the allocentric cognitive map model, and the egocentric updating model.

\section{Multiple-representation models}

On the basis of the three single-representation models, different multiple-representation models have been proposed. These multiple-representation models all assume that multiple spatial representations of a given target coexist simultaneously, and these representations are used under different circumstances. For example, a two-representation system may contain the combination of a precise egocentric updating system and a coarse enduring representation, with the assumption that the spatial updating operates on egocentric representations and, when the egocentric updating system fails, the observer switches to using the coarse enduring representation (Burgess, 2006; Mou et al., 2006; Waller \& Hodgson, 2006; Wang \& Spelke, 2000). This model will be referred to as the egocentric-updating-and-switch hypothesis.

A true egocentric-updating-and-switch hypothesis would imply a fundamental change in the spatial updating process itself, from relying on a dynamic egocentric representation

\footnotetext{
${ }^{6}$ An "and" neuron has a unique place field when encoding three or more egocentric distances of noncollinear targets. A neuron encoding the difference in the egocentric directions between two targets and the egocentric distance to one of them also has a unique place field.

${ }^{7}$ These neurons do not necessarily compute spatial locations of individual targets directly and may derive information from a separate spatial representation, such as a dynamic egocentric representation of individual target vectors.
} 
to relying on an enduring map after disorientation occurs. A related but fundamentally different hypothesis is also available. That is, the spatial-updating system always operates on transient, egocentric representations. However, the source of these egocentric representations may come directly from perception, which the observer acquires during the learning stage, or may come indirectly from long-term memory retrieval. According to this hypothesis, spatial updating operates from perceptually acquired target locations at the beginning. When interruption occurs, spatial information from an enduring representation may be "downloaded" to the spatial-updating system and converted to a new set of egocentric coordinates. ${ }^{8}$ This new egocentric representation replaces the interrupted/deteriorated one and serves as the starting point of a new round of egocentric updating process. This model may be called the egocentric-updating-andreload hypothesis. The distinction between the switch and reload hypotheses has not been made explicitly in the literature. However, these hypotheses make clearly different and testable predictions on performance after disorientation and may be a subject for future research.

There are also hypotheses that assume different types of representations for different types of targets-for example, objects versus environmental geometry (Wang \& Spelke, 2000) and random versus regular arrays (Xiao et al., 2009). However, these models are not multiple representations of the same items, and therefore, these do not necessarily coexist simultaneously. The chunking model proposed by Sargent et al. (2010) is based on a distinction between different levels of a spatial hierarchy, which is a unique form of dual representations in different scales. This chunking hypothesis makes the same predictions as the egocentric updating model when the configuration error calculation includes targets from multiple chunks. However, the effect would be diluted and harder to demonstrate when a subset of targets are grouped into chunks and not updated individually.

\section{Theoretical interpretations}

A summary of the basic and multiple-representation models is presented in Table 1. On the basis of the properties of these models, an increase in configuration errors after disorientation would reject the traditional egocentric snapshot model and the allocentric cognitive map model and would support the egocentric-updating-with-residual hypothesis, egocentricupdating-and-switch hypothesis, and egocentric-updatingand-reload hypothesis. Among these models, the egocentricupdating-with-residual hypothesis is a single-representation model and, therefore, the simplest.

\footnotetext{
${ }^{8}$ The downloading process and the lookup process are considered basically equivalent mathematically.
}

Among the dual-representation models, the simplest one is the egocentric-updating-and-reload model in which an egocentric updating system is paired with an enduring egocentric snapshot representation. That is, an egocentric-updating-andreload model has an enduring representation component, which could be either egocentric or allocentric. An egocentric-updating-and-reload model with an enduring egocentric representation is simpler than an egocentric-updatingand-reload model with an enduring allocentric representation, because the former has only one type of representation (for both its dynamic and enduring components), while the latter has two types of representations (dynamic egocentric and enduring allocentric). Similarly, an egocentric-updating-andswitch model also has an enduring component that can be either egocentric or allocentric, and the egocentric version is simpler than the allocentric version for the same reason. In addition, an egocentric-updating-and-reload model with two egocentric components is simpler than an egocentricupdating-and-switch model with two egocentric components, because the former has a single type of spatial-updating process, while the latter has two types of spatial-updating processes (updating a dynamic egocentric representation and updating self-position). Thus, among the two types of models (four versions total), an egocentric-updating-and-reload model with an enduring egocentric representation is the simplest.

In contrast, a constant configuration error rejects the egocentric-updating-with-residual hypothesis. However, all other models remain valid, and the results cannot differentiate between the traditional enduring map models (either egocentric snapshot representation or allocentric cognitive map), the egocentric-updating-and-switch hypothesis, and the egocentric-updating-and-reload hypothesis, assuming that the enduring representations are as high fidelity as the dynamic representation (e.g., an assumption made by Mou et al., 2006). Among these models, both the egocentric snapshot model and the allocentric cognitive map model are single-representation models and, therefore, the simplest. Among the dualrepresentation models, the egocentric-updating-and-reload model composed of two egocentric systems, one dynamic and one enduring, is again the simplest.

Finally, configuration errors may decrease after disorientation. A genuine decrease in the configuration errors that is not due to some other artifacts in the experimental conditions is not predicted by any of the models discussed above. For example, configuration errors may decrease due to the difference in test perspectives (Holmes \& Sholl, 2005) or due to the amount of response conflict (Waller \& Hodgson, 2006) and, therefore, cannot be interpreted on the basis of the theoretical assumptions discussed above. Such results would require an assumption that a coarse, low-quality representation is used during spatial updating, while a more precise representation becomes available when people are disoriented. The rationale behind such a system is unclear. 
Table 1 Comparison between basic and multiple-representation models

\begin{tabular}{lllll}
\hline Model & Representation type & Updating type & Number of representations & Disorientation effect \\
\hline Egocentric snapshot & Enduring & Self & 1 & no \\
Allocentric cognitive map & Enduring & Self & 1 & no \\
Egocentric updating with residual & Dynamic & Targets & 1 & yes \\
Egocentric updating and switch & Dynamic switch to enduring & Targets switch to self & 2 & Either \\
Egocentric updating and reload & Dynamic, reset by enduring & Targets & 2 & Either \\
\hline
\end{tabular}

These theoretical analyses suggest that the configuration error paradigm can be used to prove the presence of an egocentric updating system (either as a single system or as part of a dual-representation system) or to reject the traditional enduring representation models (egocentric snapshot or allocentric cognitive map), if the experimental data show an increase in configuration errors. It can also be used to reject the egocentric-updating-with-residual model, if the experimental data show constant configuration errors after disorientation. However, this paradigm cannot be used to prove the existence of allocentric cognitive maps, nor can it be used to differentiate between egocentric snapshots and allocentric cognitive maps. Thus, if the research goal is to test whether the long-term spatial memory is egocentric or allocentric, different research paradigms are needed.

It is also worth noting that despite the large difference in the rationale and methodology, experimental findings from different paradigms (e.g., the novel-shortcut, the landmark shift, the perspective taking, etc.) are often cited as converging evidence for a given type of spatial representation. This practice is questionable, however. Although, in all these approaches, spatial representations are classified as either egocentric or allocentric, the definition of these concepts are not always consistent across different paradigms. For example, a view-dependent representation may encode the object's direction and distance relative to the animal's perspective and, therefore, be considered egocentric according to the view-dependence approach. However, the information about the direction and distance of a target would allow the animal to navigate to the target directly through a straight line, even if the animal has never traveled along such a path before. Thus, such representations can be used to support novel shortcut tasks and would be classified as allocentric (survey knowledge) according to the route versus survey knowledge approach.

Similarly, some allocentric representations in the landmark-shift paradigm may be classified as egocentric according to their dependence on observer movements. For example, if an animal encodes during learning that "the redcylinder is $6 \mathrm{~m}$ to my right and the blue triangle is to the left of the cylinder" and "the feeder is $8 \mathrm{~m}$ behind me" (see Fig. 2a), it will be able to search for the feeder in relation to the landmarks during testing regardless of where the landmarks are placed, if it uses the memory of landmark locations to reorient/ relocate itself in space relative to its previous position (e.g., if the cylinder is now $12 \mathrm{~m}$ to its left and the triangle is to the right of the cylinder, the animal must have been moved $6 \mathrm{~m}$ farther away from the cylinder and turned $180^{\circ}$; therefore, the feeder must now be $10 \mathrm{~m}$ in front of it toward the left; see Fig. 2b). This reorient-then-localize process allows an animal to locate a target in relation to a landmark(s), and therefore, according to the landmark-shift paradigm, the animal uses allocentric representations. However, according to the observer-dependence approach, these representations are all egocentric. Because of the inconsistency (and even incompatibility) in the definition of spatial reference frames across different theoretical approaches and research paradigms, one should be very cautious when making generalizations from one approach to another.

\section{Empirical findings}

The empirical results using the configuration error paradigm suggested that both an increase in configuration errors after disorientation and a constant configuration error may have been observed, under different circumstances. In general, an increase in configuration error after disorientation has been shown for a randomly placed, irregular array of objects or multiple chunks of objects, while a constant configuration error has been observed for special types of targets, such as the corners of a room, parts of a single object or objects of a group, regular object layout, and imagined environment. For example, Mou et al. (2006) tested people's pointing responses to multiple targets after a single body turn (updating condition) and after disorientation while varying the learning position (either outside the object array or amid the targets) and the type of object array (regular arrangement or randomly placed). They found that the configuration errors were larger in the disorientation condition than in the updating condition when participants learned amid a random array of objects but were similar with the regular layout. Xiao et al. (2009) replicated the basic layout regularity effect and showed that configuration errors increased after disorientation for irregular layouts but not for regular layouts, regardless of whether the observer studied outside or amid the array. 
Fig. 2 An example of target localization based on landmark information
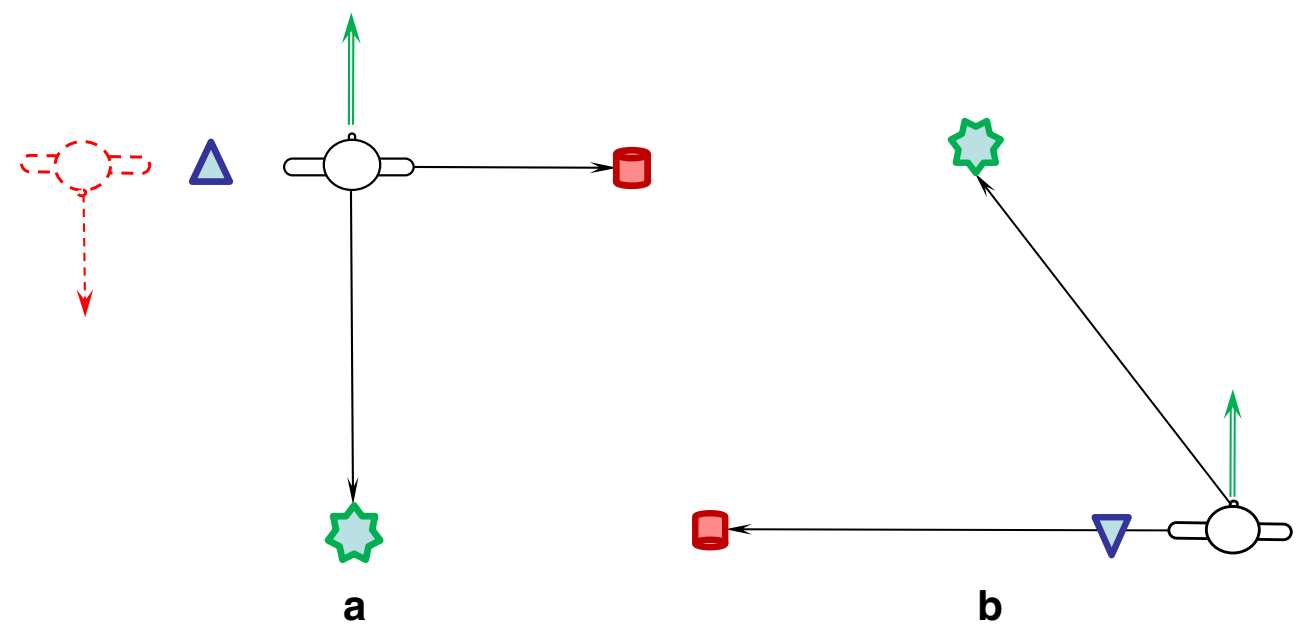

The constant configuration error before and after disorientation was sometimes interpreted as suggesting a high-fidelity allocentric representation (or enduring representation) for regular layouts. This reasoning is questionable, however, because if the participants had only a crude, low-fidelity allocentric map and used the map to localize targets both before and after disorientation (i.e., the traditional allocentric cognitive map model), the configuration error would remain constant. Thus, constant configuration error does not necessarily mean highfidelity representations, unless one makes the assumption that a high-fidelity, dynamic egocentric representation is used before disorientation and an enduring representation is used after disorientation (i.e., the egocentric-updatingand-switch model). Moreover, the experimental results also do not support this hypothesis. For example, in Xiao et al. (2009), the sizes of configuration errors for regular layouts (e.g., Experiment 2) were at least as high as those for the irregular layouts, suggesting that the representations for regular layout were not necessarily of higher fidelity than the irregular layouts.

Nevertheless, theoretically spatial localization of regular layouts is expected to be more accurate than that of irregular layouts, because of additional constraints placed on the target locations. For example, categorical knowledge about the symmetry, colinearity, and proximity can all be used to constrain the responses, which could reduce the errors. The benefit of extra categorical constraints works for both allocentric cognitive maps and egocentric representations. For example, the egocentric coordinates of one target can determine the egocentric coordinates of its symmetrical partner according to the egocentric coordinates of the symmetry axes. Thus, these additional constraints from categorical knowledge in regular arrays can help to preserve some of the relationships among the objects, as chunking/object grouping does, and therefore, in principle, will reduce the configuration error effect and make it more difficult to observe.
Xiao et al. (2009) further examined the effect of spatialupdating strategies by explicitly instructing participants to keep track of self-to-object relations when turning among a regular object array and to keep track of object-to-object relations while turning among an irregular object array. The results showed that the effect of disorientation could be moderated by the instructions. Configuration errors increased after disorientation for the regular array when self-to-object instructions were given and remained constant for the irregular object array when object-to-object instructions were given, but only when participants learned from outside the array. These results suggest that spatial updating can be influenced by top-down factors such as motivation or attention (Amorim, Glasauer, Corpinot, \& Berthoz, 1997).

However, note that object-to-object relation does not necessarily mean allocentric cognitive map (or enduring representation in general) and does not necessarily mean constant configuration errors. For example, the instruction to "keep track of all the object-to-object relations" used in Xiao et al. (2009) implies that the directional relation is encoded in an egocentric frame. If the object-to-object vector is encoded in an external directional frame, the coding does not change as the observer moves, and there is nothing to keep track of. That is, one needs to keep track of the object-to-object relationships only if they are expressed with respect to the observer's heading and, therefore, are egocentric by definition. According to the egocentric updating model, when multiple object-to-object relations are encoded relative to the observer's heading and updated individually, there is no guarantee that the internal consistency across different object-to-object relation codes can be preserved, for the same reason that the internal consistency across a set of individually updated egocentric vectors should be disrupted by disorientation. Accordingly, if participants in their studies followed the instructions faithfully, they should actually show an increase in configuration errors after disorientation. Thus, the interobject relationship theory cannot explain the experimental results on the instruction effect. 
A more plausible explanation of the effect of object-toobject instruction comes from the chunking hypothesis proposed by Sargent et al. (2010). Because it is unlikely that participants are able to perform the instructed task (i.e., keeping track of all pairs of object-to-object relations), due to the capacity limitations in the spatial-updating system, participants may have interpreted the instruction as grouping all targets as a "chunk" and updating the chunk as an individual object. Because objects within a chunk are not updated individually, according to Sargent et al. (2010), they are immune to the effect of disorientation, resulting in a constant configuration error before and after disorientation. This hypothesis also provides a possible explanation on the effect of learning position, because chunking should be easier if participants had experience from the outside perspective, where all targets were clustered within a small visual field, than from the among-the-objects perspective, where the targets were all dispersed in space. Thus, the instruction effect can be explained by chunking of self-toobject vectors, without assuming a separate interobject relation system.

Waller and Hodgson (2006) tested participants' pointing responses either to multiple targets in their immediate surroundings or to objects in their bedroom while imagining being in the middle of their bedroom. They found that the configuration errors increased after disorientation for targets in their immediate surroundings, but not for those in a remote, familiar environment (e.g., their bedroom at home). These results support an egocentric updating process for immediate environments, but not for imagined environments retrieved from long-term memory.

In addition, Waller and Hodgson (2006) found that configuration errors remained constant for blindfolded turning angles between $0^{\circ}$ and $90^{\circ}$ and then increased abruptly for the turning angle of $135^{\circ}$, which was not significantly different from the disorientation condition in the other experiments. These results were interpreted as an abrupt switch from egocentric updating to relying on an enduring representation. However, the experimental finding of an abrupt increase in configuration errors is controversial. For example, in Xiao et al. (2009), three levels of configuration errors were observed (Experiments 3, 4, 5, 6, 8), with the disorientation condition higher than the updating condition $\left(240^{\circ}\right.$ rotation), which was higher than the baseline condition $\left(0^{\circ}\right.$ rotation). These findings are inconsistent with the hypothesis of a single switch after a $>90^{\circ}$ turning. How configuration errors change exactly during spatial updating still awaits further research, and such research needs to address the methodological challenge that the testing perspectives cannot be matched for different turning angles, which can potentially introduce artifacts in the configuration error measure that may confound the theoretical interpretations.
Sargent et al. (2010; Sargent et al., 2008) tested participants' pointing responses to a set of target objects after a succession of turns before being fully disoriented and used the angular separation error (ASE, similar to the configuration error) to examine whether the internal relationship between targets was preserved over the turning and disorientation. They found evidence that the ASE showed larger increase for some subset of objects than for others after turning and disorientation. For example, the ASE was smaller for targets closer to each other than for those farther apart. ASE was also smaller for targets forming a collinear array than for those in a random array and for targets belonging to parts of a single object than for those on different objects. These findings support the hierarchical model of spatial representations and an egocentric updating process operating at certain levels of the spatial hierarchy, while the subordinate level spatial information (i.e., withinchunk spatial information) is not directly updated. The chunking hypothesis is comparable with both egocentric and allocentric enduring representations. In essence, chunking means that a set of objects are not updated individually and only a single vector representing the set is updated. In fact, the traditional enduring representation models (egocentric snapshot and allocentric cognitive map) can be considered special cases of the chunking hypothesis, where all targets are grouped into a single "chunk." Thus, the logic for the traditional enduring representations can all be applied to the representation of the elements within an individual chunk.

Some controversial results were presented by Holmes and Sholl (2005), who measured the configuration errors of pointing responses to multiple targets in both novel and familiar environments. They found comparable configuration errors in the eyes-closed turning condition and the disorientation condition. These findings were interpreted as evidence for allocentric location codes, which are immediately available in a novel, room-sized environment in addition to familiar environments. However, these results were inconsistent with those in other studies (Mou et al., 2006; Waller \& Hodgson, 2006; Wang \& Spelke, 2000; Xiao et al., 2009). Several speculations were made on the potential difference in methodology - for example, large turning angle (Waller \& Hodgson, 2006), relatively regular array (Mou et al., 2006) - and it was noted that, as compared with other studies, the configuration errors were very high even before disorientation. The exact reason of the discrepancy is not clear.

\section{Conclusions}

Research has generally shown that configuration errors in pointing to multiple groups of targets or targets from an irregular array increase after disorientation (although see Holmes \& Sholl, 2005). These results rejected the traditional 
allocentric cognitive map and egocentric snapshot representations as the mechanism underlying object localization during observer movements. However, other models are all consistent with the existing experimental evidence.

There is also evidence that configuration errors in pointing to certain types of targets (e.g., corners of a room, regular arrays, targets retrieved from long-term memory, parts of a single object, etc.) are not affected by disorientation. These results rejected the egocentric-updating-with-residual hypothesis as an explanation of the mechanism underlying spatial localization of targets with some specific properties. In contrast, both the egocentric snapshot model and the allocentric cognitive map model predict the constant configuration error results. Moreover, the dual-representation hypotheses with an egocentric updating system coupled with an enduring representation are also consistent with the experimental findings.

Taken together, there are two models that can accommodate both phenomena - that is, the egocentric-updating-andswitch hypothesis and the egocentric-updating-and-reload hypothesis. The egocentric-updating-and-reload hypothesis requires a single type of spatial updating process and, therefore, is the simpler of the two models. Within the egocentricupdating-and-reload hypothesis, the version with an enduring egocentric component is simpler than the version with an enduring allocentric component, because the former has one type of spatial representations (egocentric for both dynamic and enduring components), while the latter has two types of spatial representations. Moreover, in general, if a primitive form of representation is sufficient to explain the experimental results, these data cannot be taken as evidence that an animal possesses a more advanced form of representation, and the primitive representation should be taken as the preferred explanation. Since egocentric representations are generally considered the more primitive form of spatial representations, the simplest interpretation of the existing findings using the configuration error paradigm is a version of the egocentric-updating-and-reload model, which has an enduring egocentric snapshot representation component.

Author Note Thanks to David Waller for suggestions on an earlier draft of the manuscript. Some of the content was presented at the 52nd Annual Meeting of the Psychonomic Society, Seattle, WA, 2011. Comments and questions should be addressed to Frances Wang (francesw@cyrus.psych.illinois.edu).

\section{References}

Amorim, M. A., Glasauer, S., Corpinot, K., \& Berthoz, A. (1997). Updating an object's orientation and location during nonvisual navigation: A comparison between two processing modes. Perception \& Psychophysics, 59, 404-418.

Bennett, A. T. D. (1996). Do animals have cognitive maps? Journal of Experimental Biology, 199, 219-224.
Burgess, N. (2006). Spatial memory: How egocentric and allocentric combine. Trends in Cognitive Sciences, 10, 551-557.

Burgess, N., Spiers, H. J., \& Paleologou, E. (2004). Orientational manoeuvres in the dark: Dissociating allocentric and egocentric influences on spatial memory. Cognition, 94, 149-166.

Cartwright, B. A., \& Collett, T. S. (1982). How honey bees use landmarks to guide their return to a food source. Nature, 295, 560 564.

Chan, G. S. W., Chang, Y., \& Sun, H. J. (2007). Encoding of different environmental features with or without spatial updating. Journal of Vision, 7(9), 1053.

Collett, T. S., \& Cartwright, B. A. (1983). Eidetic images in insects: Their role in navigation. Trends in Neurosciences, 6, 101-105.

Collett, T. S., \& Lehrer, M. (1993). Looking and learning a spatial pattern in the orientation flight of the wasp vespula-vulgaris. Proceedings of the Royal Society of London B, 252, 129-134.

Collett, T. S., \& Rees, J. A. (1997). View-based navigation in Hymenoptera: Multiple strategies of landmark guidance in the approach to a feeder. Journal of Comparative Physiology. A, 181, 47-58.

Easton, R. D., \& Sholl, M. J. (1995). Object-array structure, frames of reference, and retrieval of spatial knowledge. Journal of Experimental Psychology: Learning, Memory, and Cognition, 21, 483500 .

Eichenbaum, H., \& Cohen, N. J. (1988). Representation in the hippocampus: What do hippocampal neurons code? Trends in Neurosciences, 11, 244-248.

Eichenbaum, H., Otto, T., \& Cohen, N. J. (1992). The hippocampuswhat does it do? Behavioral and Neural Biology, 57, 2-36.

Endress, A. D., \& Wood, J. N. (2011). From movements to actions: Two mechanisms for learning action sequences. Cognitive Psychology, 63, 141-171.

Gallistel, C. R. (1990). The organization of learning. Cambridge, MA: MIT Press.

Gleason, T. C., \& Rothblat, L. A. (1994). Landmark discrimination in the rat: A measure of allocentric spatial ability. Behavioral Neuroscience, 108, 206-209.

Gould, J. L. (1986). The locale map of honey bees: Do insects have cognitive maps? Science, 232, 861-863.

Griffin, A. S., \& Etienne, A. S. (1998). Updating the path integrator through a visual fix. Psychobiology, 26, 240-248.

Holmes, M. C., \& Sholl, M. J. (2005). Allocentric coding of object-toobject relations in overlearned and novel environments. Journal of Experimental Psychology: Learning, Memory, and Cognition, 31, 1069-1087.

Klatzky, R. L. (1998). Allocentric and egocentric spatial representations: Definitions, distinctions, and interconnections. In C. Freksa, C. Habel, \& K. F. Wender (Eds.), Spatial cognition: An interdisciplinary approach to representing and processing spatial knowledge (pp. 117). Berlin: Springer.

Knierim, J. J., Kudrimoti, H. S., \& McNaughton, B. L. (1995). Place cells, head direction cells, and the learning of landmark stability. Journal of Neuroscience, 15, 1648-1659.

Landau, B., Spelke, E. S., \& Gleitman, H. (1984). Spatial knowledge in a young blind child. Cognition, 16, 225-260.

Lehrer, M., \& Collett, T. S. (1994). Approaching and departing bees learn different cues to the distance of a landmark. Journal of Comparative Physiology. A, 175, 171-177.

Maguire, E. A., Spiers, H. J., Good, C. D., Hartley, T., Frackowiak, R. S. J., \& Burgess, N. (2003). Navigation expertise and the human hippocampus: A structural brain imaging analysis. Hippocampus, $13,250-259$.

Mallot, H. A., \& Gillner, S. (2000). Route navigating without place recognition: What is recognised in recognition-triggered responses? Perception, 29, 43-55.

McNaughton, B. L., Knierim, J. J., \& Wilson, M. A. (1995). Vector encoding and the vestibular foundations of spatial 
cognition: Neurophysiological and computational mechanisms. In M. Gazzaniga (Ed.), The cognitive neurosciences (pp. 585595). Cambridge, MA: MIT Press.

Meilinger, T. (2008). The network of reference frames theory: A synthesis of graphs and cognitive maps. In C. Freksa, N. S. Newcombe, P. Gärdenfors, \& S. Wölfl (Eds.), Spatial cognition $V I$ (pp. 344-360). Berlin: Springer.

Mou, W., \& McNamara, T. P. (2002). Intrinsic frames of reference in spatial memory. Journal of Experimental Psychology: Learning, Memory, and Cognition, 28, 162-170.

Mou, W., McNamara, T. P., Rump, B., \& Xiao, C. (2006). Roles of egocentric and allocentric spatial representations in locomotion and reorientation. Journal of Experimental Psychology: Learning, Memory, and Cognition, 32, 1274-1290.

O'Keefe, J., \& Nadel, L. (1978). The hippocampus as a cognitive map. Oxford: Oxford University Press, Clarendon Press.

O'Keefe, J., \& Speakman, A. (1987). Single unit activity in the rat hippocampus during a spatial memory task. Experimental Brain Research, 68, 1-27.

Sargent, J., Dopkins, S., Philbeck, J., \& Chichka, D. (2010). Chunking in spatial memory. Journal of Experimental Psychology: Learning, Memory, and Cognition, 36, 576-589.

Sargent, J., Dopkins, S., Philbeck, J., \& Modarres, R. (2008). Spatial memory during progressive disorientation. Journal of Experimental Psychology: Learning, Memory, and Cognition, 34, 602-615.

Shelton, A. L., \& McNamara, T. P. (2001). Systems of spatial reference in human memory. Cognitive Psychology, 43, 274-310.

Simons, D. J., \& Wang, R. F. (1998). Perceiving real-world viewpoint changes. Psychological Science, 9, 315-320.

Tolman, E. C. (1948). Cognitive maps in rats and men. Psychological Review, 55, 189-208.
Waller, D., \& Hodgson, E. (2006). Transient and enduring spatial representations under disorientation and self-rotation. Journal of Experimental Psychology: Learning, Memory, and Cognition, 32, $867-882$.

Wang, R. F. (2003). Spatial representations and spatial updating. In D. E. Irwin \& B. H. Ross (Eds.), The psychology of learning and motivation, 42: Advances in research and theory. Cognitive vision (pp. 109-156). San Diego, CA: Academic Press.

Wang, R. F. (2007). Spatial processing and view-dependent representations. In F. Mast \& L. Jancke (Eds.), Spatial processing in navigation, imagery, and perception (pp. 49-65). New York: Springer.

Wang, R. F., \& Brockmole, J. R. (2003). Human navigation in nested environments. Journal of Experimental Psychology: Learning, Memory, and Cognition, 29, 398-404.

Wang, R. F., \& Simons, D. J. (1999). Active and passive scene recognition across views. Cognition, 70, 191-210.

Wang, R. F., \& Spelke, E. S. (2000). Updating egocentric representations in human navigation. Cognition, 77, 215-250.

Wang, R. F., \& Spelke, E. S. (2002). Human spatial representation: Insights from animals. Trends in Cognitive Sciences, 6, 376-382.

Wang, R. F., \& Spelke, E. S. (2003). Comparative approaches to human navigation. In K. Jeffery (Ed.), The neurobiology of spatial behaviour (pp. 119-143). Oxford: Oxford University Press.

Wilson, M. A., \& McNaughton, B. L. (1993). Dynamics of the hippocampal ensemble code for space. Science, 261, 1055-1058.

Xiao, C., Mou, W., \& McNamara, T. P. (2009). Use of self-to-object and object-to-object spatial relations in locomotion. Journal of Experimental Psychology: Learning, Memory, and Cognition, 35, $1137-1147$. 